\title{
Lipid disorders in HIV patients: what about raised HDL- Cholesterol?
}

\author{
Satyajit Das* \\ Integrated Sexual Health Service and Clinical Assessment Service, Coventry \& Warwickshire Partnership Trust, UK
}

The lipid disorders seen in individuals with HIV infection include elevated triglycerides (TG) and total cholesterol (TC), a decrease in high-density lipoprotein cholesterol (HDL-C), and variable effects on low-density lipoprotein (LDL) cholesterol. The exact mechanism is still not clear, and the cause could be multifactorial. The individual contributions of HIV infection, specific antiretroviral agents, host genetics and changes in body composition, all should be considered. However, there has been change in the pattern of dyslipidaemia in HIV patients. This is because most of the newer antiretrovirals have better lipid profile, either they are lipid neutral or causes mild dyslipidaemia. Raised lipids have been observed less often and low HDL-C has been observed less often as well. The Multicentre AIDS Cohort Study (MACS) showed that immediately after HIV infection TC and HDL-C dropped and when treatment is started TC goes back to normal or above normal level and HDL-C comes back to normal or near normal [1]. We wonder whether HDL-C can go above normal or even abnormally high in some patients when they are on treatment for a long time. Raised HDL-C has been observed with nevirapine use but is not known to be associated with an abnormally high level. Abnormally high HDL-C has been noted in HIV patients, but the actual prevalence of abnormally high HDL-C is not reported.

HDL Cholesterol (HDL-C) is known to be cardio-protective. Several large epidemiological studies demonstrated that low HDL-C can be an independent predictor of increased risk of cardiovascular disease (CVD). Increasing HDL-C levels in animals has shown markedly reduced susceptibility to the development of arteriosclerosis in animal models. However, the interventional studies increasing the level of HDL-C in humans is yet to demonstrate any beneficial effect on the outcome of CVD. There is evidence that some gene variants that raises the HDL cholesterol level may not be accompanied by a decreased risk of having a cardiovascular event. Recent results from two population-based studies in Copenhagen involving over 100,000 people demonstrated that all-cause mortality is high in men and women with increased levels of HDL-C [2]. Similar findings were observed in a Canadian observational cohort study that included over 630,000 people and the participants were followed for about five years [3]. This study demonstrated that the risk of developing CVD in both men and women were higher when their HDL-C level were either in the lower range or at the very highest levels, compared to the levels which were in the intermediate range. In both men and women, the effect of high HDL-C and increased risk of death was higher in the raised HDL-C group (Figure 1).

Obviously, these studies raised questions about the protective effect of HDL-C at extreme levels either very low or very high. It appears that the HDL-C level instead of having a linear relationship with the risk of cardiovascular disease, it appears to have a ' $U$ ' shape relationship, where the two arms of ' $U$ ' represents either the lower HDL-C or higher HDL-C level (Figure 1).

The function of HDL-C is complex, however some of them are wellknown and they are related to efflux of cholesterol from the macrophages, reverse cholesterol transport, antioxidant and anti-inflammatory effect on blood vessels [4]. Reverse cholesterol transport is a process by which cholesterol is transferred from peripheral tissues and cells to the liver and kidney. Free cholesterol effluxed from macrophages are taken up by apolipoprotein-A (apo-A) and form HDL cholesterol which is then transferred back to the liver or excreted through the kidney (Figure 2). Impaired cholesterol efflux capacity has been found to be an important reason for low HDL cholesterol in HIV patients particularly when they are not on antiretroviral treatment. HIV within the macrophages can inhibit ATP binding cassette (ABC) transporter, a key enzyme for cholesterol efflux from macrophages and keep the cholesterol within the macrophages. With antiretroviral treatment the macrophages tend to clear from HIV and cholesterol efflux starts resulting in normalisation of plasma HDL-C level.

The plasma level of HDL-C may not reflect the actual function of HDL-C. There are several different types of HDL-C and depends upon their cholesterol content and the size of the HDL cholesterol (Figure 2). There has been debate throughout on the ability of HDL-C to have its function when the cholesterol content within the particle is high or low. Probably it is more important to see the number of HDL particles rather than the actual concentration of HDL-C.

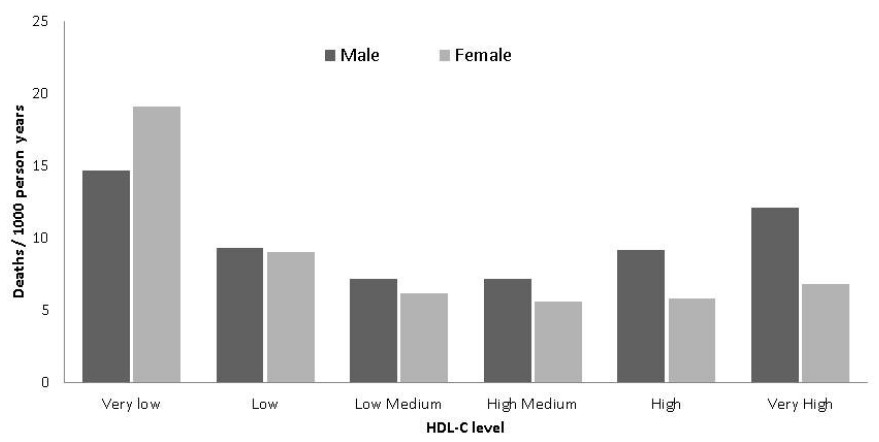

Figure 1. Relationship between crude all-cause mortality rate and HDL-C level [3]. Very low HDL-C $<0.7 \mathrm{mmol} / \mathrm{L}$, Very high HDL-C level $>2.3 \mathrm{mmol} / \mathrm{L}$

${ }^{*}$ Correspondence to: Satyajit Das, Professor of Sexual Health and HIV, Integrated Sexual Health Service and Clinical Assessment Service, Coventry \& Warwickshire Partnership Trust, UK, E-mail: Satyajit.das@covwarkpt.nhs.uk

Received: February 08, 2019; Accepted: February 15, 2019; Published: February 18,2019 


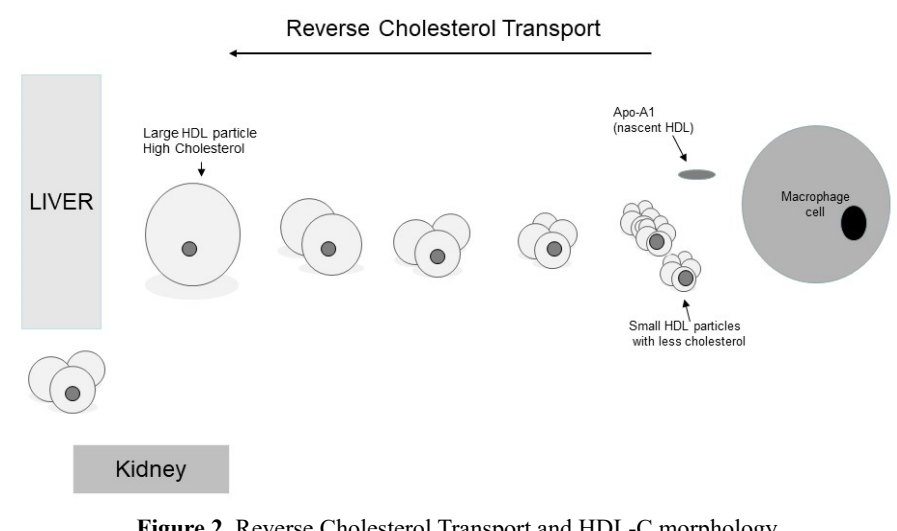

The risk of CVD in HIV patients has been found to be relatively higher compared to the general population. Some observational cohort studies found a link between abacavir use and Myocardial Infarction which has been disputed by other studies $[5,6]$. The national guidelines [7] recommend that patients who have an increased risk of CVD should avoid using abacavir. The algorithms which assess the risk of cardiovascular disease, like Framingham Risk Calculation or Q-Risk Calculation, all use total cholesterol and HDL (TC/HDL) ratio and patients with abnormally raised total cholesterol along with raised HDL-C may give a total TC/HDL ratio within the normal range. This may under-estimate the risk of cardiovascular disease. In this context it becomes more important to see whether this HDL-C has got the functional ability to prevent arteriosclerosis. However, assessment of HDL-C function in clinical practice is far from reality at present.

It is important that we have understanding about the magnitude of problem HIV patients have with abnormally high HDL-C level and underlying any risk factor associated with that. In the absence of any clarity about the interventions we need to take to deal with abnormally raised HDL-C we need to continue to manage known modifiable cardiovascular risk factors. At present our aim has been to reduce total cholesterol level and increasing HDL-C level and we considered different satins which can reduce cholesterol at the same time can increase HDL-C levels. Research in the general population and also in the HIV population gave priority to use drugs which can lower total cholesterol levels and increase HDL-C level. We did not have to consider whether we need to lower abnormally high HDL-C level. In fact, we are not sure which should be our priority when individuals present with abnormally raised HDL-C with raised total cholesterol level. This appears to be a different scenario and we do not have any clarity about what interventions to use.

Hence in the absence of any definite guidance about how to deal with abnormally raised HDL-C we should aim to focus on assessment and modification of other risk factors like smoking, hypertension, insulin resistance and raised total cholesterol level. Choice of ARV regimens should be individualized for the patient to achieve maximal and durable viral suppression as well as avoiding long-term toxicities. It would be reasonable to target raised total cholesterol levels with lipid lowering agents, which is been the case at present in the general population, for HIV patients.

\section{References}

1. Riddler SA, Smit E, Cole SR, Li R, Chmiel JS, et al. (2003) Impact of HIV infection and HAART on serum lipids in men. JAMA 289: 2978-2982. [Crossref]

2. Madsen CM, Varbo A, Nordestgaard BG (2017) Extreme high high-density lipoprotein cholesterol is paradoxically associated with high mortality in men and women: two prospective cohort studies. Eur Heart J 38: 2478-2486. [Crossref]

3. Ko DT, Alter DA, Guo H, Koh M, Lau G, et al. (2016) High-Density Lipoprotein Cholesterol and Cause-Specific Mortality in Individuals Without Previous Cardiovascular Conditions: The CANHEART Study. J Am Coll Cardiol 68: 2073-2083. [Crossref]

4. Fisher EA, Feig JE, Hewing B, Hazen SL, Smith JD (2012) High-density lipoprotein function, dysfunction, and reverse cholesterol transport. Arterioscler Thromb Vasc Biol 32: 2813-20. [Crossref]

5. Friis-Møller N, Sabin CA, Weber R, d'Arminio Monforte A, El-Sadr WM, et al. (2003) Combination antiretroviral therapy and the risk of myocardial infarction. $N$ Engl J Med 349:1993-2003. [Crossref]

6. Das S (2010) Risk of cardiovascular disease in HIV-infected patients. J Antimicrob Chemother 65: 386-389. [Crossref]

7. Angus B, Awosusi F, Das S, Brook G et al. (2019) BHIVA Guidelines for the routine investigation and monitoring of HIV-1 positive individulas.

Copyright: (C2019 Satyajit D. This is an open-access article distributed under the terms of the Creative Commons Attribution License, which permits unrestricted use, distribution, and reproduction in any medium, provided the original author and source are credited. 